\title{
Long non-coding RNA Linc00205 promotes hepatoblastoma progression through regulating microRNA-154-3p/Rho-associated coiled-coil Kinase 1 axis via mitogen-activated protein kinase signaling
}

\author{
Guoqing Liư ${ }^{1}$, Qiang Zhu ${ }^{1}$, Hao Wang ${ }^{1}$, Jianfeng Zhou ${ }^{1}$, Bin Jiang ${ }^{1}$ \\ ${ }^{1}$ Department of General Surgery, Children's Hospital of Nanjing Medical University, Nanjing 210008, China
}

Correspondence to: Bin Jiang; email: jiangbin363@163.com, https://orcid.org/0000-0002-4176-0693

Keywords: hepatoblastoma, miR-154-3p, ROCK1, MAPK signaling pathway

Received: February 1, $2021 \quad$ Accepted: September 18, $2021 \quad$ Published: February 17, 2022

Copyright: (C) 2022 Liu et al. This is an open access article distributed under the terms of the Creative Commons Attribution License (CC BY 3.0), which permits unrestricted use, distribution, and reproduction in any medium, provided the original author and source are credited.

\begin{abstract}
Hepatoblastoma (HB) is the most common pediatric liver tumor. The significant tumor heterogeneity of HB leads to varied prognoses among children with the disease. Recent studies have suggested that long non-coding RNAs (IncRNAs) can serve as novel therapies for HB treatment. Thus, in this study, we aimed to reveal the function and mechanism of the IncRNA Linc00205 in HB. Our results exhibited that, in both HB tissues and cell lines, levels of Linc00205 were significantly increased. In addition, knockdown of Linc00205 led to suppression of HB development. Moreover, we identified that Linc00205 was able to directly bind to miR-154-3p, thus isolating miR-154-3p from its target Rho-associated coiled-coil Kinase 1 (ROCK1). Further cellular behavioral experiments elucidated that the miR-154-3p inhibitor and ROCK1 overexpression were able to reverse the effect of downregulated Linc00205 on proliferation, migration, invasion, and apoptosis of HB cells by rescue assays via mitogen-activated protein kinase (MAPK) signaling. Our results demonstrated that Linc00205 enhanced HB progression by regulating ROCK1 expression via sponging miR-154-3p through MAPK signaling, which suggests a novel potential therapeutic target for HB.
\end{abstract}

\section{INTRODUCTION}

Hepatoblastoma (HB) is the most common primary malignant tumor of the liver among children, as it accounts for over $1 \%$ of pediatric malignancies. In recent years, there has been an increase in the incidence of $\mathrm{HB}$, which is thought to be related to a higher number of premature births. Furthermore, infants with a birth weight lower than $1500 \mathrm{~g}$ are predisposed to developing HB [1]. HB presents as a solid focal tumor that reproduces the liver in an embryologic development state [2]. The primary curative treatment for $\mathrm{HB}$ is surgical excision, and chemotherapy is essential in order to shrink an unresectable tumor to becoming resectable, and decreasing surgery-related morbidity [3]. Children that are within the low-risk category can have an 80\% 5-year survival rate after treatment. However, for children that are high risk or after a relapse, the survival rate decreases to $30-40 \%$ [4]. Therefore, there is a significant need to achieve a more detailed understanding of HB in order to develop more effective therapies to improve patient outcomes.

Long non-coding RNAs (lncRNAs) are RNAs that are longer than 200 nucleotides. As they are non-coding RNAs, lncRNAs do not possess an open reading frame that encodes protein [5]. LncRNAs are known to regulate a variety of biological processes, including cell proliferation and differentiation, through scaffolding protein-protein/DNA interaction, binding to protein or microRNAs (miRNAs), or enhancers of genes. As lncRNAs can have an effect on cell cycle, dysregulation of IncRNAs can be found across different types of cancers. Studies have suggested that IncRNAs can play a role in cancer initiation and progression [6]. Recently, long intergenic non-protein coding RNA 205 
(Linc00205) have been found to promote malignancy in lung cancer by recruiting the RNA-binding protein FUS to stabilize CSDE1 mRNA, which enhances lung cancer cell proliferation, apoptosis, and migration [7].

MiRNAs are another class of non-coding RNAs, but are only 18-25 nucleotides in length. MiRNAs are known to regulate gene expression by binding target messenger RNAs (mRNAs) at the 3'UTR in order to initiate degradation or repression of translation [8]. On the other hand, miR-4510 was considered to be a tumor suppressor as it suppressed the $\mathrm{Wnt} / \beta$-catenin pathway by targeting the glypican-3 (GPC3) oncogene, thereby decreasing $\mathrm{HB}$ cell line and HuH6 cell viability [9]. miR-154-3p is tightly related to numerous physiological and pathological processes, including diabetes [10], cardiac complications [11] and cancers [12]. However, the function of miR-154-3p in $\mathrm{HB}$ remains unclear.

The relationship between IncRNAs and miRNAs has been explored across many studies. The lncRNATUG1 was found to be upregulated in HB tissues and cell lines, and interact with miR-34a-5p as a sponge in order to inhibit mRNAs from binding to its targets. The target mRNA of miR-34a-5p is VEGFA, which is a crucial protein that is involved in pathological angiogenesis. Thus, TUG1 promotes tumor growth and angiogenesis in $\mathrm{HB}$ by preventing miR-34a-5p from regulating VEGFA, which can cause TUG1 to be a possible therapeutic target for HB [13]. Linc00205 has been recognized as a molecular sponge for miR-122-5p in HCC cell line to promote cell growth [14]. However, the concrete role of Linc00205 in $\mathrm{HB}$ remains to be investigated for deep understanding.

Rho-associated coiled-coil kinases (ROCKs) are serine/threonine kinases that are downstream effectors of the small GTPases RhoA, RhoB, and RhoC. There are two ROCKs, including ROCK1 and ROCK2, which are similar to each other. Depending on their cellular location, they can function differently by regulating cell proliferation, apoptosis, cell adhesion, motility, and contraction [15]. Many studies have focused on the role of ROCKs in cancer. Several somatic mutations in ROCK1 are present in human cancers, and all these mutations have enhanced kinase activity of ROCK1, as well as increased motility of tumor cells [16]. MiRNAs are able to regulate ROCK1 expression, and many miRNAs expression changes have been identified in cancer tissues. The miR-124 and miR-145 were both found to target ROCK1 in glioma cells, and inhibit glioma migration $[17,18]$. Furthermore, in thyroid cancer cells, ROCK1 expression was found to be negatively correlated to miR-154-3p [19].

Herein, the function and related mechanisms of lncRNA Linc00205 in HB were explored in-depth, and the results may be valuable for HB therapy.

\section{RESULTS}

\section{Linc00205 expression is significantly increased in $\mathrm{HB}$}

We performed qRT-PCR assays in order to determine the expression of Linc00205 in HB tissues and paracancerous normal tissues collected from 60 patients. The expression of Linc00205 in HB tissues was found to be remarkably higher compared to adjacent nontumor tissues (Figure 1A; $* * * p<0.001$ ). In addition, increased Linc00205 expression was observed in the three HB cell lines (Figure 1B; ***p $<0.001, * * \mathrm{p}<0.01$, $\left.{ }^{*} \mathrm{p}<0.05\right)$. Furthermore, Kaplan-Meier survival analyses indicate that among the 60 patients, patients with low Linc00205 expression in their HB tissues were found to have a much higher five-year survival rate after surgery (Figure 1C, $\mathrm{p}=0.036$ ). The results indicated that
A

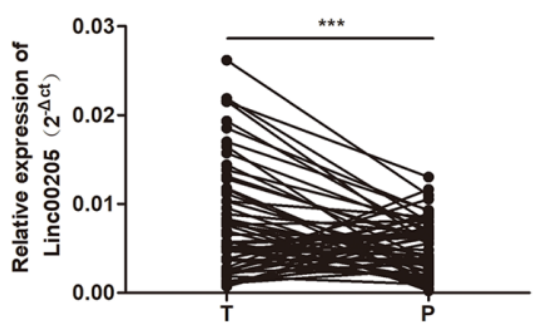

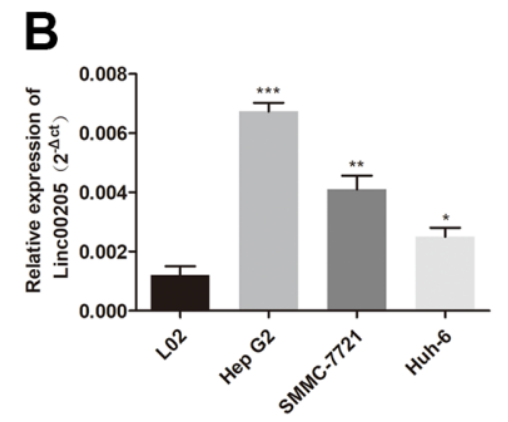

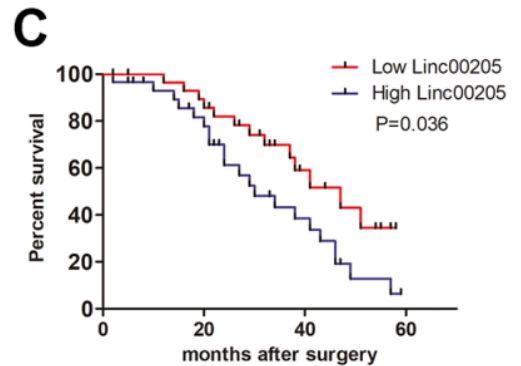

Figure 1. Linc00205 expression in HB increased. (A) qRT-PCR assay for Linc00205 expression among HB ( $n=60$ ) and para-cancerous tissues $(n=60)$. (B) qRT-PCR assay for Linc00205 expression in HB cell lines. (C) Kaplan-Meier curve for survival after surgery with low or high Linc00205 expression. ${ }^{*} p<0.05, * * p<0.01, * * * p<0.001$. 
dysregulation of Linc00205 expression may play an important role in HB malignancy.

\section{Regulating Linc00205 expression can have an effect cell viability, proliferation, apoptosis, migration, invasion, and tumor formation}

In order to further investigate the role of Linc00205 in $\mathrm{HB}$, we downregulated Linc00205 by transfecting HepG2 cells using si-Linc00205, as HepG2 cells were found to have the highest Linc00205 expression across all $\mathrm{HB}$ cell lines. We also upregulated Linc00205 by transfecting Huh-6 cells with Linc00205 ov, as Huh-6 cells have the lowest Linc00205 expression across all HB cell lines. Next, we utilized qRT-PCR assays to determine transfection efficiency (Figures 2A, 3A, $* p<0.05, * * p<0.01$ ). The CCK-8 assays exhibited that, in comparison with the negative controls, cell viability was found to decrease with Linc00205 knockdown (Figure 2B, *p<0.05), and increased with Linc00205 overexpression (Figure $3 \mathrm{~B}, * \mathrm{p}<0.05)$. Furthermore, we found that fewer colonies formed after knockdown of Linc00205 (Figure 2C, $* * \mathrm{p}<0.01$ ) and more colonies had Linc00205 overexpression (Figure 3C, **p<0.01) through colony formation assays. Flow cytometry results indicated that apoptotic rate increased when we downregulated Linc00205 (Figure 2D, **p<0.01), and decreased when Linc00205 was overexpressed (Figure 3D, $* * p<0.01$ ). Both transwell migration assays and wound healing assays led to an impairment in cell migration in Linc00205 knockdown cells (Figure 2E, 2G, **p<0.01), as well as enhanced expression in Linc00205 overexpression cells (Figure $3 \mathrm{E}, 3 \mathrm{G}, * * \mathrm{p}<0.01)$. The transwell invasion assay verified that knockdown of Linc00205 impaired cell invasion (Figure $2 \mathrm{~F}, * * \mathrm{p}<0.01$ ), while overexpression of Linc00205 enhanced cell invasion (Figure 3F, $* * p<0.01)$. These results suggest that Linc00205 is a promoter of HB malignancy and suppressed Linc00205 is likely to suppress HB progression.

We also administered a subcutaneous injection of $\mathrm{HB}$ cells that were transfected with either si-Linc00205 or Linc00205 ov to mice in order to induce tumor formation. Tumors that were induced by si-Linc 00205 transfected HepG2 cells were found to be significantly smaller in size, as well as lighter in weight, compared to tumors induced by si-NC-transfected HepG2 cells (Figure 4A, 4C, 4E). Furthermore, tumors that were induced by Linc00205 ov transfected Huh- 6 cells were significantly larger in size and heavier in weight compared to tumors that were induced by $\mathrm{NC}$ transfected Huh-6 cells (Figure 4B, 4D, 4F). Lastly, we detected expression of Linc00205 in xenograft tumors through the use of qRT-PCR assays. We found that si-Linc00205 group had lower expression of Linc00205 compared to the si-NC group (Figure 4G), while Linc00205 ov group had higher expression of Linc00205 than the NC group (Figure 4H). Therefore, Linc00205 regulation may represent a novel therapy for $\mathrm{HB}$ patients.

\section{Linc00205 inhibited miR-154-3p as a sponge and inhibition of miR-154-3p restored HepG2 cells from Linc00205 knockdown}

It has been previously reported thatLinc00205 interacts with miR-122-5p in HCC cell lines to promote growth [14]. Therefore, we explored possible Linc00205targeted miRNAs, and discovered a complementary sequence between Linc00205 and miR-154-3p (Figure 5A), which suggests that miR-154-3p may possibly be a direct target of Linc00205. The dualluciferase assay results validated this interaction, as luciferase activity was significantly reduced by pGL3Linc00205-wt and miR-154-3p co-transfection, compared to the control group (Figure $5 \mathrm{~B}, * \mathrm{p}<0.05$ ). Furthermore, qRT-PCR assay results demonstrated that expression of miR-154-3p in HB tissues was significantly downregulated, compared to the paracancerous tissues (Figure 5C, **p<0.01). Which indicates that Linc00205 can directly sponge miR-154$3 p$, and that upregulation of Linc00205 can cause downregulation of miR-154-3p, which suggests a possibility that Linc 00205 mediates HB progression by downregulating miR-154-3p.

In order to confirm the regulatory role between Linc00205 and miR-154-3p, we inhibited miR-154-3p in Linc00205-knockdown HepG2 cells. miR-154-3p expression was significantly reduced in the siLinc00205+miR-154-3p inh. Group, compared to the si-Linc00205 group (Figure 6A, p<0.01). Furthermore, cell viability significantly increased (Figure 6B, ${ }^{*} \mathrm{p}<0.05$ ), and more colonies were formed (Figure 6C, $\mathrm{p}<0.01)$ compared to the si-Linc00205 group without miR-154-3p inhibitor. The apoptosis rate was also found to be significantly decreased with miR-154-3p inhibition, compared to the si-Linc00205 group (Figure 6D, $\mathrm{p}<0.01$ ). Both the transwell migration assay and wound healing assay demonstrated cell migration (Figure 6E, 6G) and invasion ability (Figure 6F) of the Linc00205 knockdown cells, as miR-154-3p inhibition was significantly increased compared to the si-Linc00205 group. In conclusion, through the use of miR-154-3p inhibition, the malignancy that was suppressed by Linc00205 knockdown was restored. All these findings suggest that upregulated Linc00205 suppresses miR-154-3p regulatory role in $\mathrm{HB}$ cells, which causes cell carcinogenesis. 
A

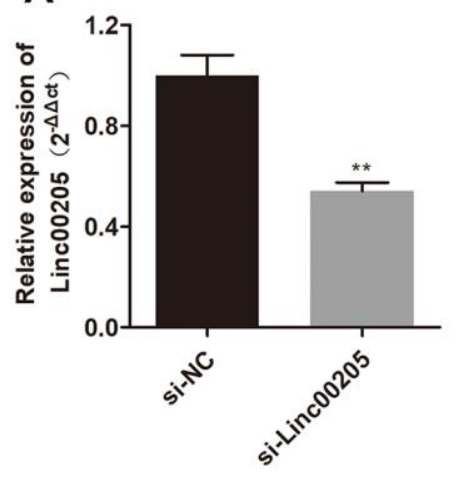

B

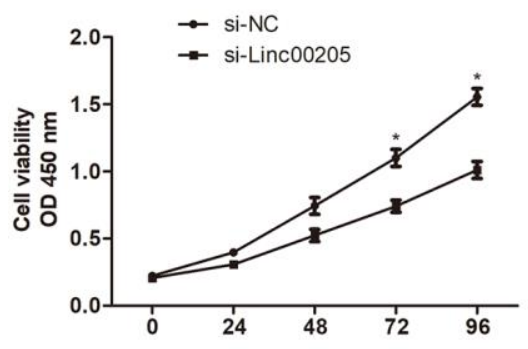

C

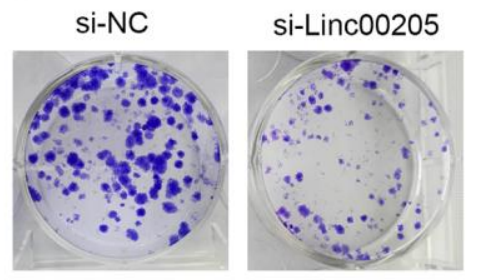

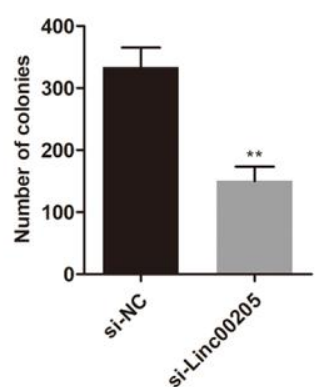

D

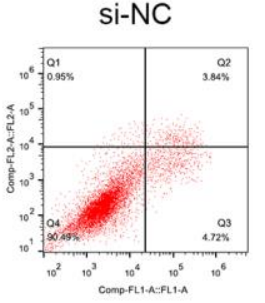

si-Linc00205

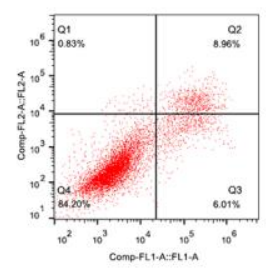

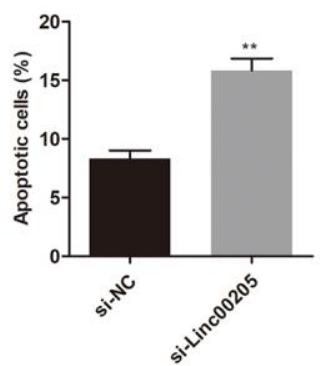

G

E

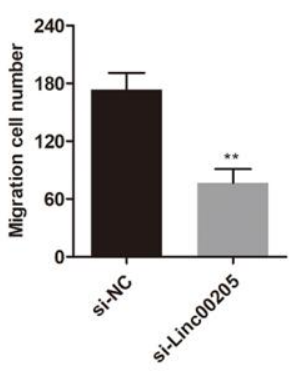

Oh
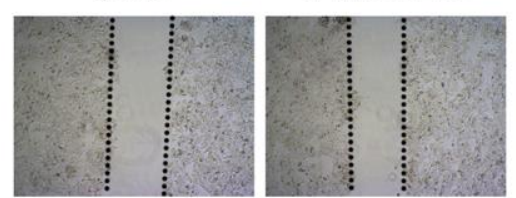

$48 \mathrm{~h}$
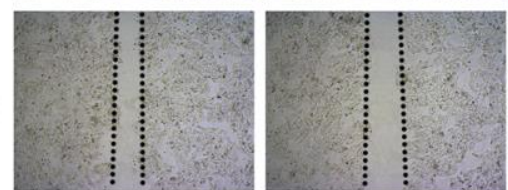

F
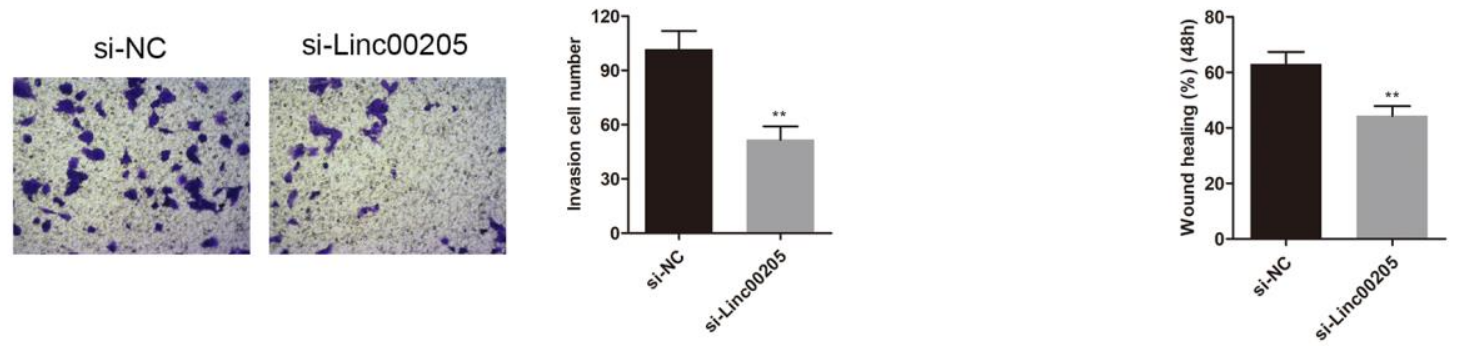

Figure 2. Knockdown of Linc00205 altered cell viability, colony formation, apoptosis, migration and invasion ability. (A) qRTPCR assay for Linc00205 expression in HepG2 cells that were transfected with either si-Linc00205 or control si-NC. (B) Cell viability assay conducted in HepG2 cells after transfection with either si-Linc00205 or control si-NC. (C) Colony formation ability of HepG2 cells that were transfected with si-Linc00205 or control si-NC. Quantitative analysis of colonies formation (right panel). (D) Apoptosis of HepG2 cells transfected with si-Linc00205 or control si-NC, and quantitative analysis of the apoptosis cells (right panel). (E, F) The migration and invasion ability of HepG2 cells that were transfected with either si-Linc00205 or control si-NC, as determined using the transwell migration assay (E) and transwell invasion assay (F) (left panel). Quantitative analysis of the migratory cells or invasive cells in transwell assays was also conducted (right panel). (G) Migration ability of HepG2 cells transfected with si-Linc00205 or control si-NC at indicated time points was evaluated by wound healing assay (upper panel). Quantitative analysis of the gap of wound healing assay (lower panel) was performed at 48 hours. ${ }^{*} p<0.05, * * p<0.01$. 
A

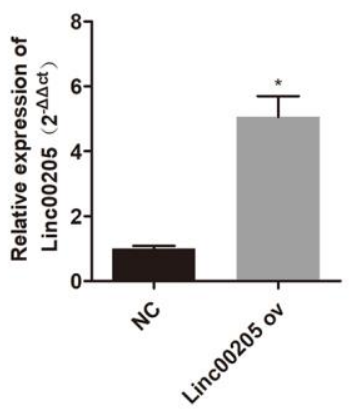

B

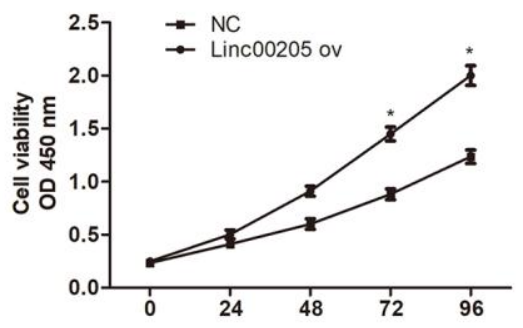

E

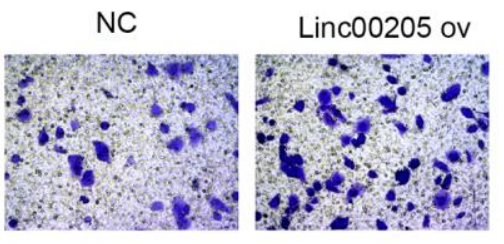

F

NC

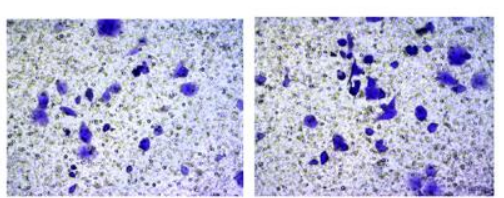

C
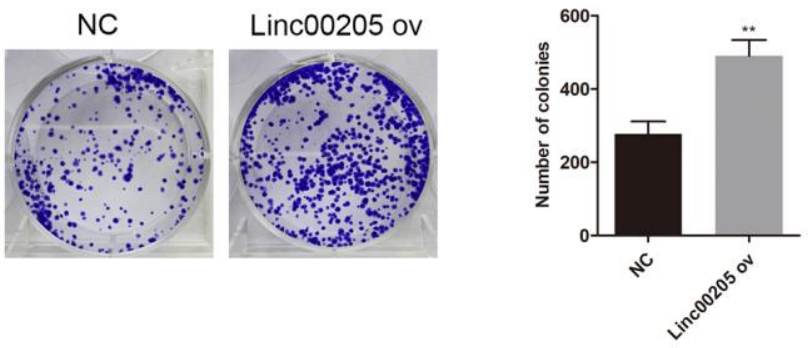

D
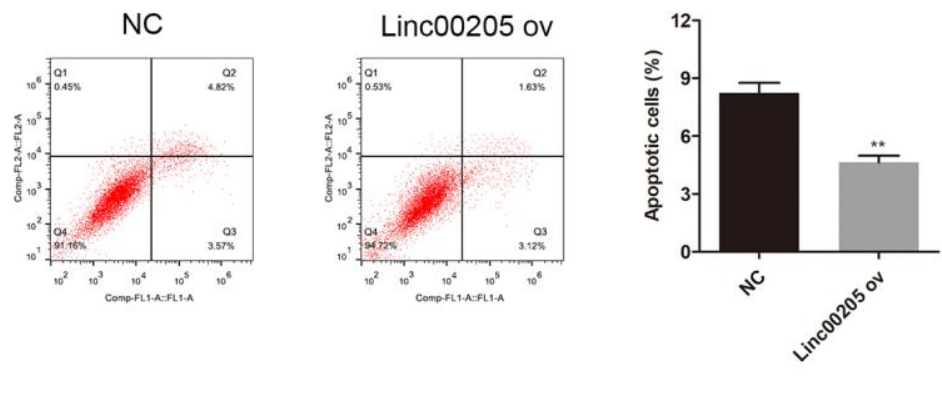

G
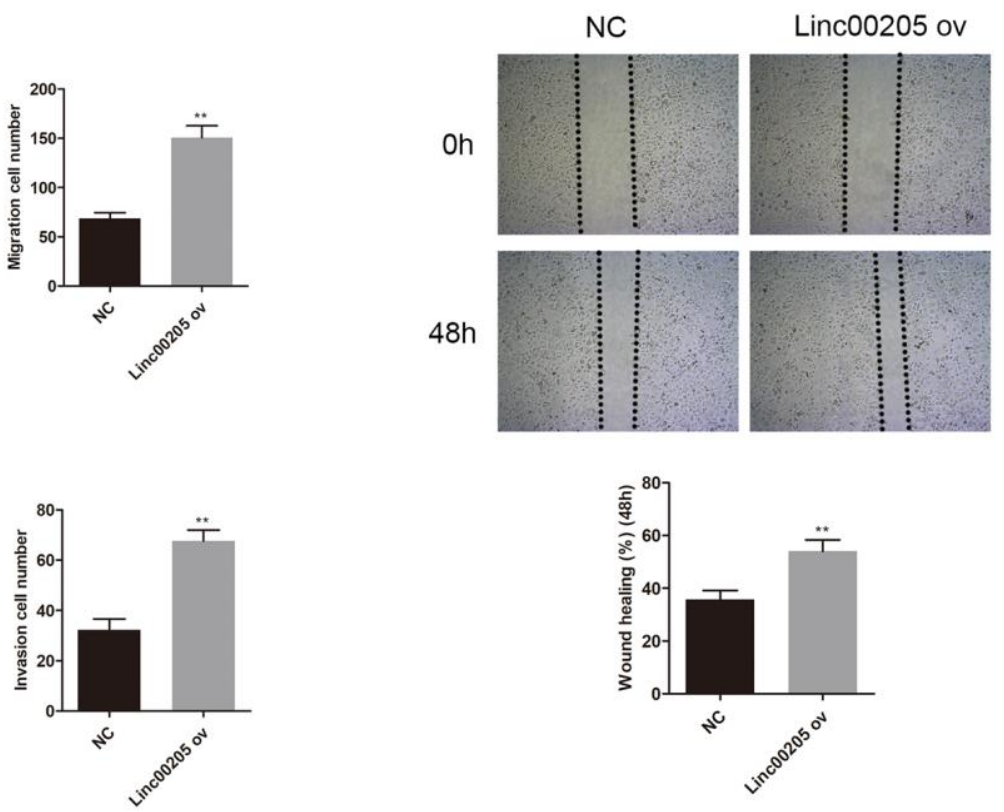

Figure 3. Overexpression of Linc00205 altered cell viability, apoptosis, colony formation ability, apoptosis, migration and invasion ability. (A) qRT-PCR assay for Linc00205 expression in Huh-6 cells transfected with Linc00205 ov expression (ov) or NC. (B) Cell viability assay was conducted in Huh- 6 cells after transfection of Linc00205 ov or NC. (C) Colony formation ability of Huh- 6 cells were transfected with either Linc00205 ov or NC. Furthermore, we conducted quantitative analysis of colony formation (right panel). (D) Apoptosis assays of Huh- 6 cells transfected with either Linc00205 ov or NC, as well as quantitative analysis of the apoptosis cells (right panel). (E, F) The migration and invasion ability of Huh- 6 cells transfected with Linc00205 ov or NC were determined through the use of a transwell migration assay (E), and transwell invasion assay (F) (left panel). Quantitative analysis of the migratory cells or invasive cells in transwell assays was also performed (right panel). (G) Migration ability of Huh-6 cells transfected with Linc00205 ov or NC at indicated time points were evaluated by wound healing assay (upper panel). Quantitative analysis of the gap of wound healing assay (lower panel) was performed at 48 hours. $* p<0.05, * * p<0.01$. 

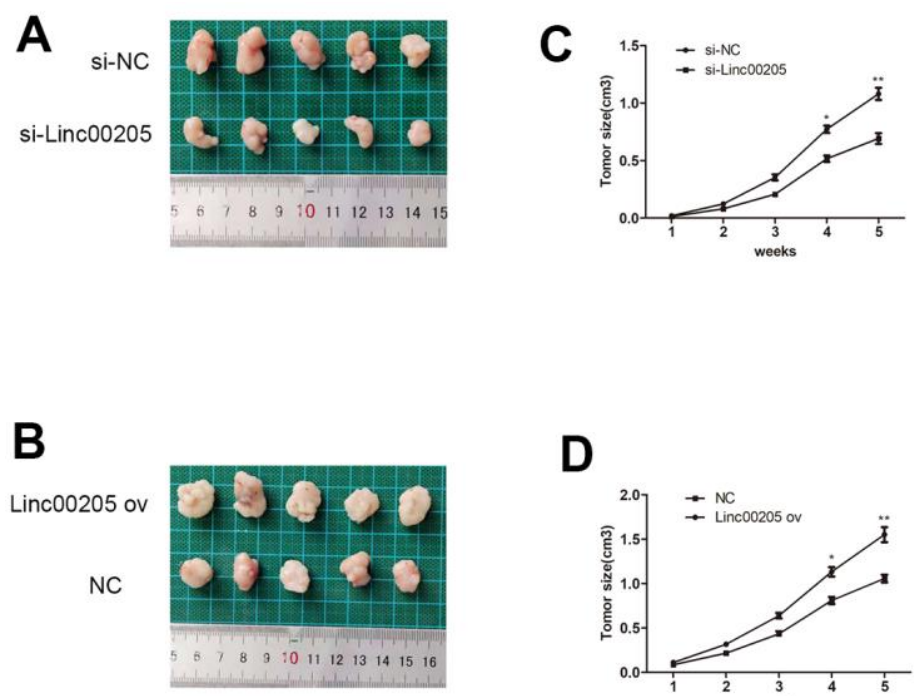
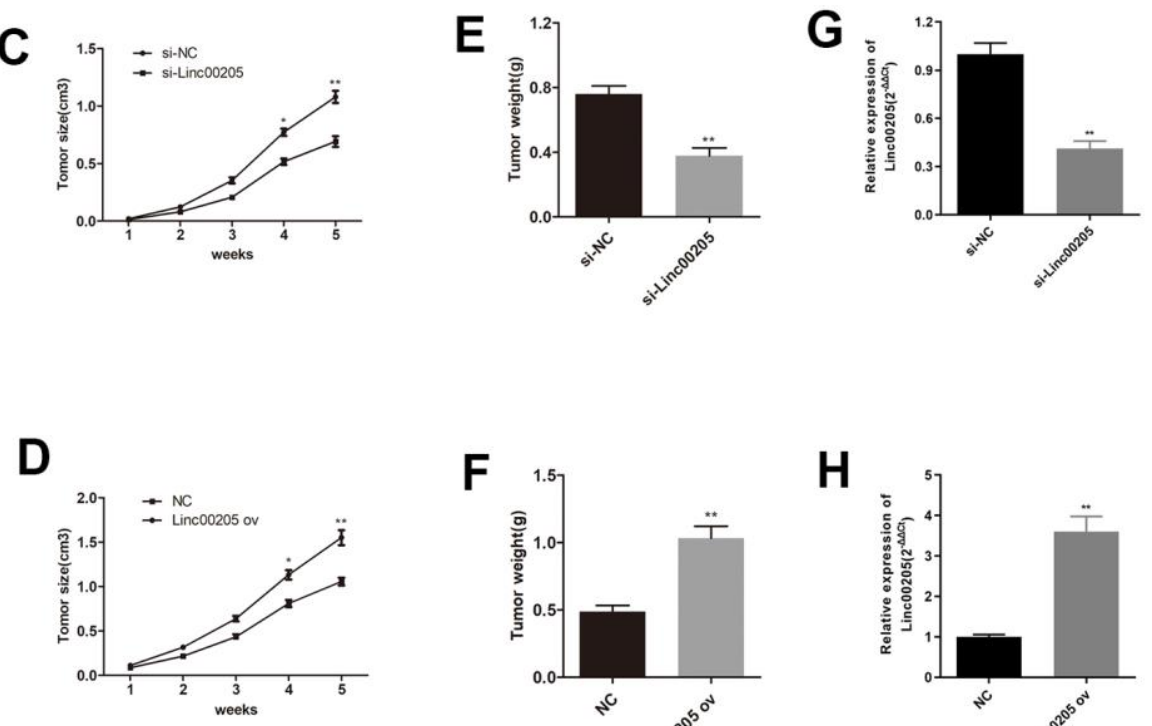
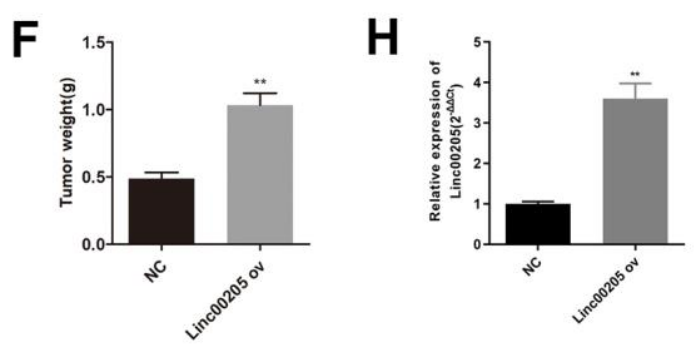

Figure 4. Linc00205 expression affects HB cell-induced tumor formation. (A, B) The representative appearances of the extracted xenograft tumors with implantation of si-Linc00205 transfected HepG2 cells (A) or Linc00205 ov transfected Huh-6 cells (B). (C, D) The size of tumors that were implanted with si-Linc00205 transfected HepG2 cells (C) or Linc00205 ov transfected Huh-6 cells (D), which were measured every seven days. (E, F) Quantitative analysis of weight of tumors with implantation of si-Linc00205 transfected HepG2 cells (E) or Linc00205 ov transfected Huh-6 cells (F). (G, H) qRT-PCR assay for Linc00205 expression in HepG2 cells that were transfected with si-Linc00205 or si-NC among xenograft tumors (G) or in Huh-6 cells transfected with Linc00205 ov or NC of xenograft tumors $(\mathbf{H}) .^{*} \mathrm{p}<0.05, * * \mathrm{p}<0.01$.

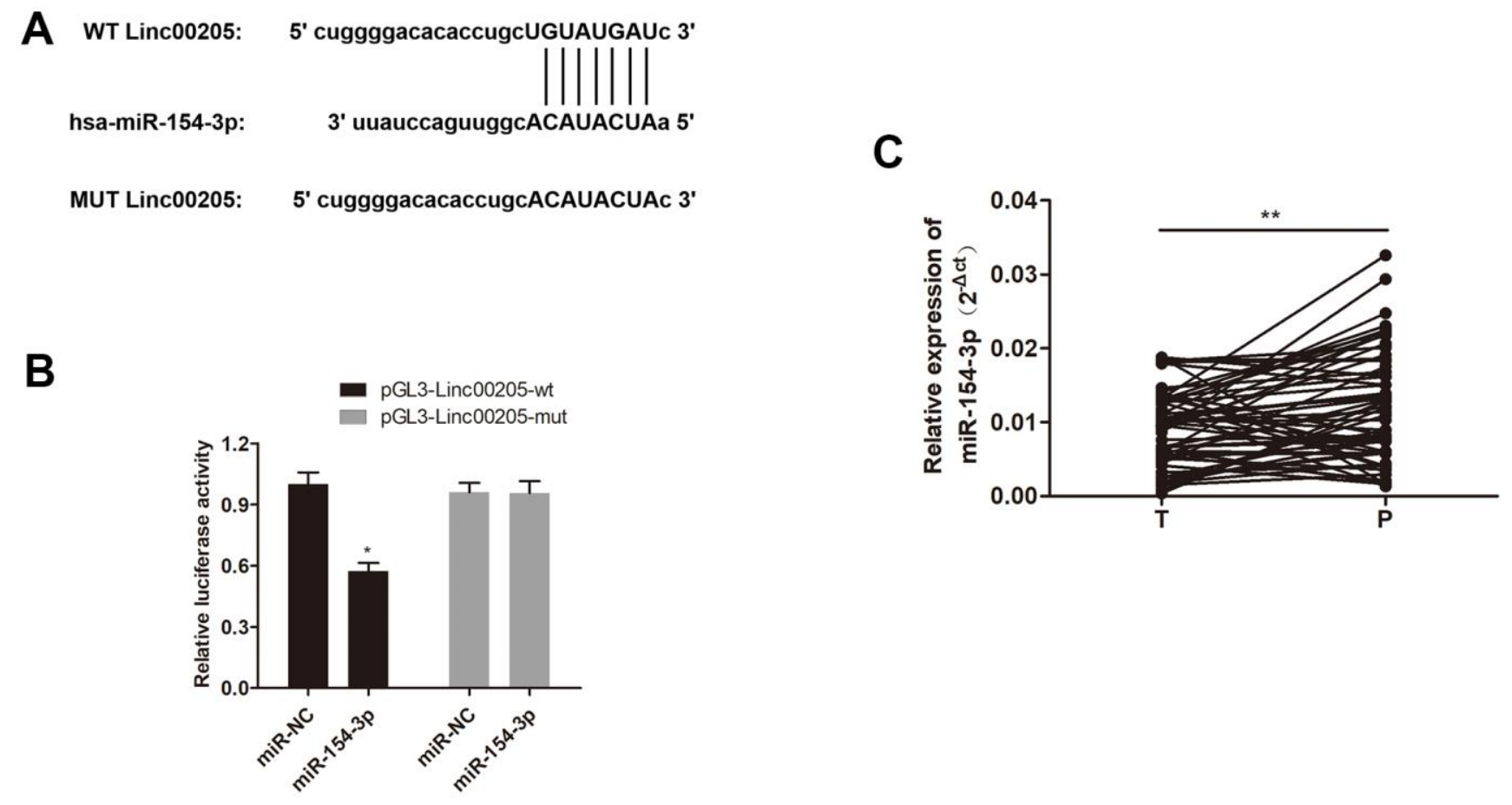

Figure 5. miR-154-3p interacts with Linc00205 in HB cells. (A) A schematic representation of the complementary sequence between Linc00205 and miR-154-3p. (B) Relative luciferase activity was examined in miR-154-3p and pGL3-Linc00205-wt or pGL3Linc00205-mut co-transfected cells. (C) The qRT-PCR assays for miR-154-3p expression in HB tissues $(n=60)$ and para-cancerous tissues. $* p<0.05, * * p<0.01$. 
A
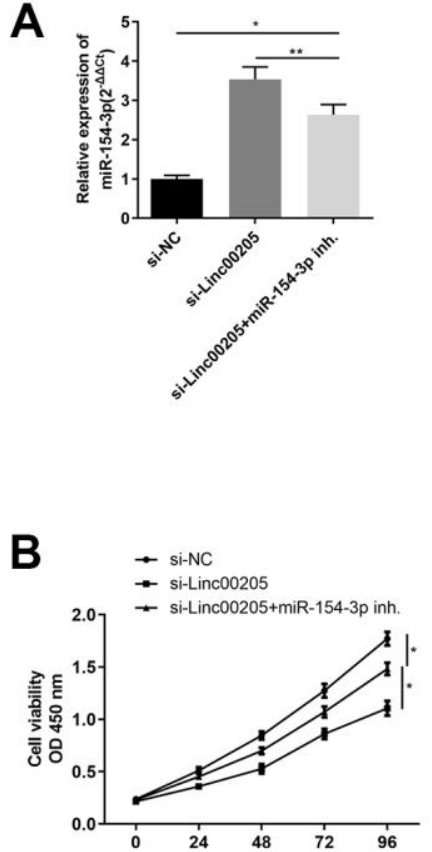

E

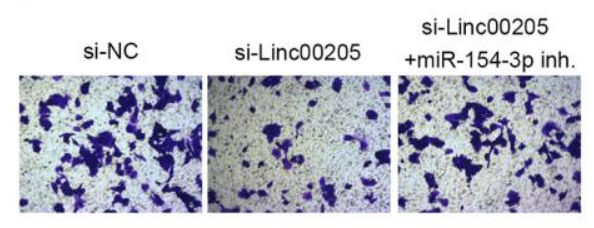

$\mathbf{F}$

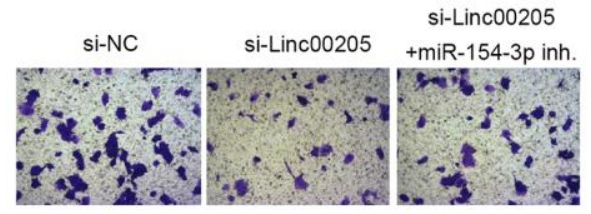

C
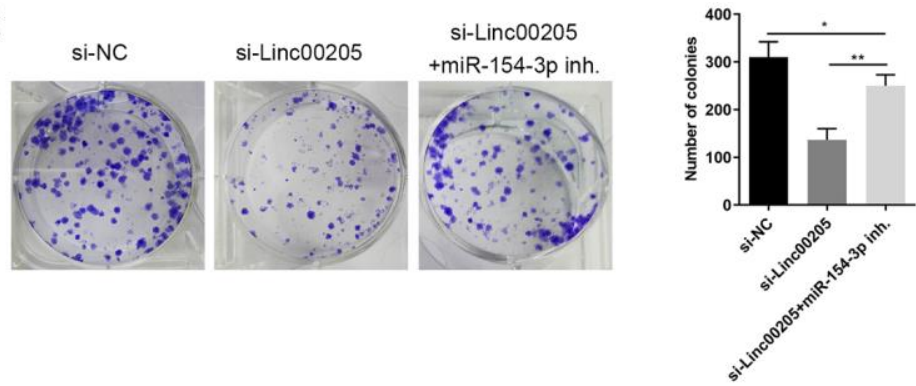

D
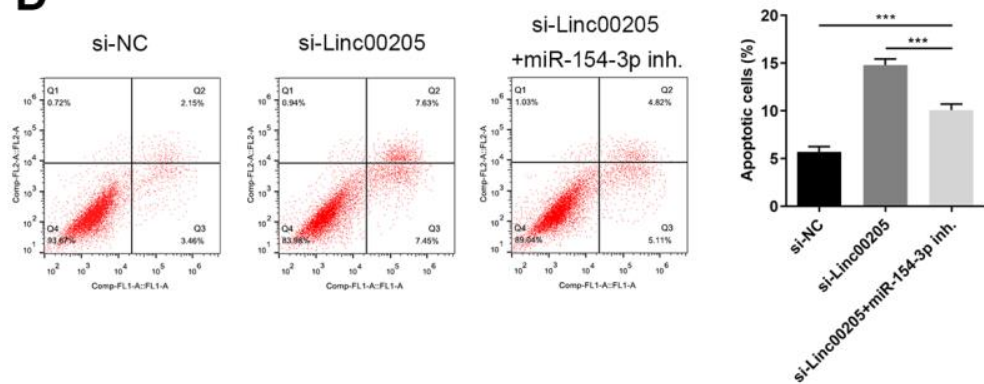

G
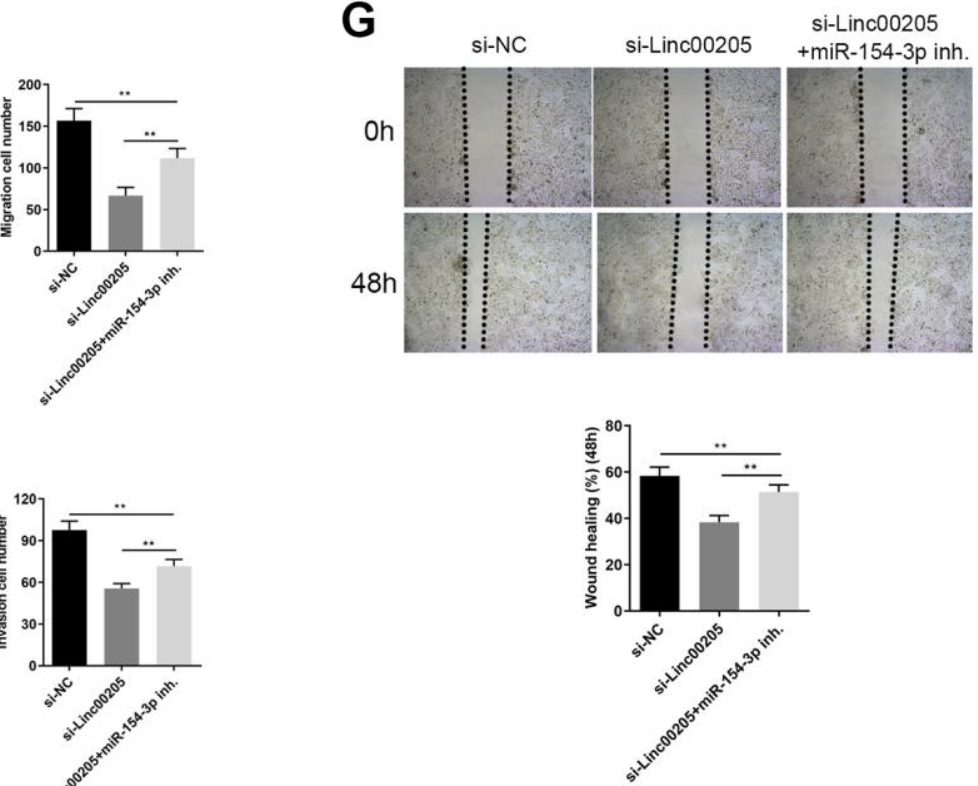

Figure 6. Inhibition of miR-154-3p reversed changes in cell viability, colony formation ability, apoptosis, migration and invasion ability, caused by Linc00205 knockdown. (A) qRT-PCR assay for miR-154-3p expression among HepG2 cells transfected with siLinc00205 and with or without miR-154-3p inhibitor. (B) Cell viability assay was carried out in HepG2 cells that were transfected with siLinc00205 and with or without the miR-154-3p inhibitor. (C) Colony formation ability of HepG2 cells were transfected with si-Linc00205 and with or without miR-154-3p inhibitor. A quantitative analysis of colonies was performed (right panel). (D) Apoptosis assays of HepG2 cells transfected with si-Linc00205 and with or without miR-154-3p inhibitor, and quantitative analysis of the apoptosis cells (right panel). (E, F) The migration and invasion ability of HepG2 cells transfected with si-Linc00205 and with or without miR-154-3p inhibitor were determined through the use of a transwell migration assay $(\mathbf{E})$ and transwell invasion assay (F) (left panel). A quantitative analysis of migratory or invasive cells in transwell assays was also performed (right panel). (G) Migration ability of HepG2 cells transfected with either si-Linc00205 and with or without miR-154-3p inhibitor at indicated time points was evaluated by wound healing assay (upper panel). The quantitative analysis of the gap of wound healing assay (lower panel) was performed at 48 hours. ${ }^{*} p<0.05$. 
ROCK1 is a target of miR-154-3p, which is dysregulated in $\mathrm{HB}$ tissues, and overexpression of ROCK1 restored HepG2 cells from miR-154-3p upregulation

Furthermore, we investigated downstream target of miR154-3p. Additionally, ROCK1 expression was found to negatively correlate with miR-154-3p [19], and a putative miR-154-3p binding site was discovered in the ROCK1 mRNA 3'UTR (Figure 7A), and significantly reduced luciferase activity was observed. Thus, we carried out a dual-luciferase assay and validated that miR-154-3p binds directly to ROCK1 by pGL3ROCK1-wt and miR-154-3p co-transfection, compared to the control group (Figure $7 \mathrm{~B},{ }^{*} \mathrm{p}<0.05$ ). In addition, qRT-PCR and western blot assays for ROCK1 mRNA expression (Figure $7 \mathrm{C}, * * * \mathrm{p}<0.001$ ) and ROCK1 protein expression (Figure 7D) in HB tissues were shown to be significantly higher than in para-cancerous tissues, which suggests ROCK1 may have a role in HB progression.

In order to investigate the regulatory relationship between ROCK1 and miR-154-3p, we transfected
HepG2 cells with a miR-154-3p mimic with or without ROCK 1 ov. Co-transfection with ROCK1 ov remarkably increased ROCK1 levels, compared to the miR-154-3p mimics group (Figure 8A, p $<0.01$ ). Results of the CCK8 assays showed that cell viability was enhanced by ROCK1 overexpression, compared to the miR-154-3p mimics group (Figure $8 \mathrm{~B},{ }^{*} \mathrm{p}<0.05$ ). Furthermore, more colonies were formed with ROCK1 overexpression in colony formation assays, compared to the miR-154-3p mimics group (Figure 8C, p<0.01). Flow cytometry results suggested that there were fewer apoptotic cells with ROCK1 overexpression, compared to the miR-154$3 p$ mimics group (Figure 8D, $p<0.01$ ). The transwell migration assay (Figure 8E, $\mathrm{p}<0.01$ ) and wound healing assay (Figure $8 \mathrm{G}, \mathrm{p}<0.01$ ) both demonstrated that the cell migration ability was enhanced with ROCK1 overexpression, compared to the miR-154-3p mimics transfected cells, and the capacity was restored to level of the mimics NC group. Invasion ability was also enhanced with ROCK1 overexpression, compared to the miR-154-3p group (Figure $8 \mathrm{~F}, \mathrm{p}<0.01$ ). These results suggest that miR-154-3p can downregulate ROCK1 function, and have an essential role in inhibiting $\mathrm{HB}$ development.
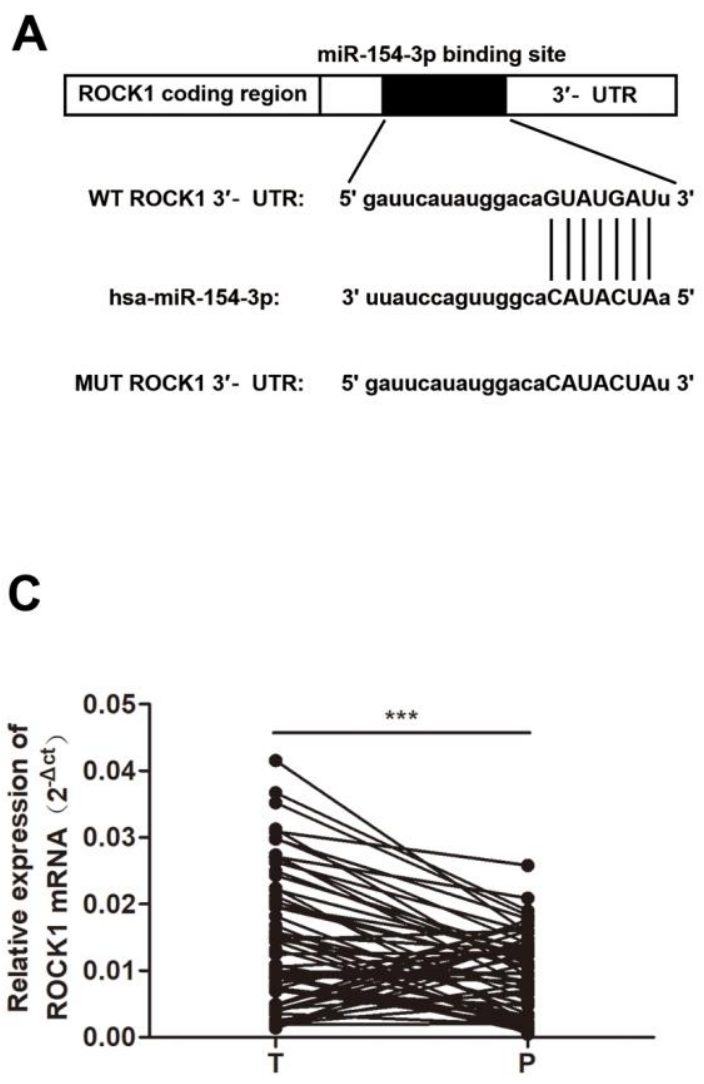
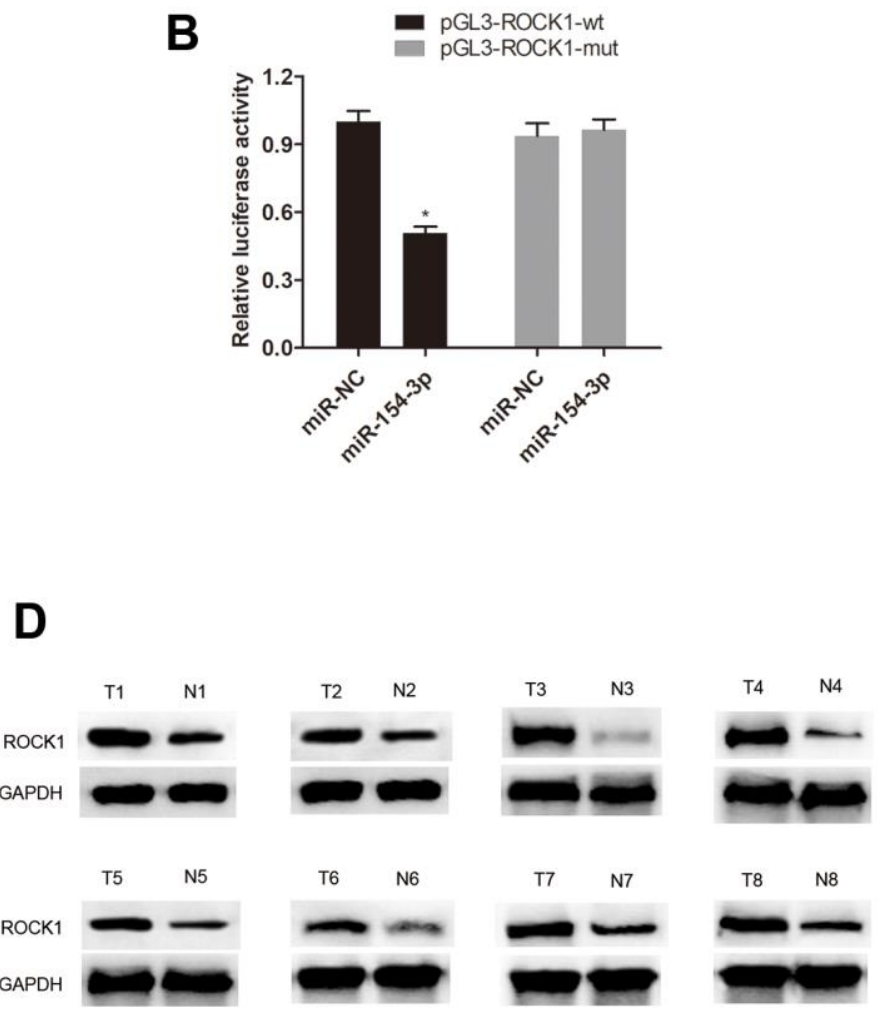

Figure 7. miR-154-3p directly targets ROCK1. (A) A schematic representation of putative miR-154-3p binding site in ROCK1. (B) Relative luciferase activity was examined in miR-154-3p and pGL3-ROCK1-wt or pGL3-ROCK1-mut co-transfected cells. (C, D) qRT-PCR and western blot assays for ROCK1 mRNA (C) $(n=60)$ and protein expression (D) $(n=8)$ in HB tissues and para-cancerous tissues. ${ }^{*} p<0.05, * * * p<0.001$. 
A

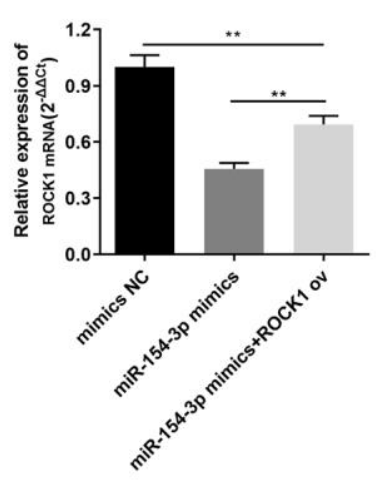

B

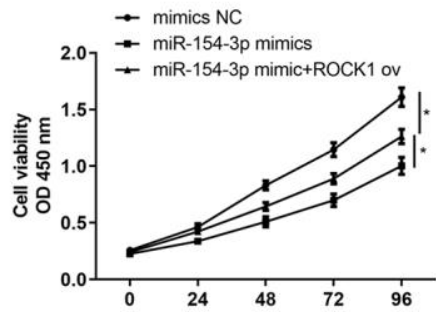

E

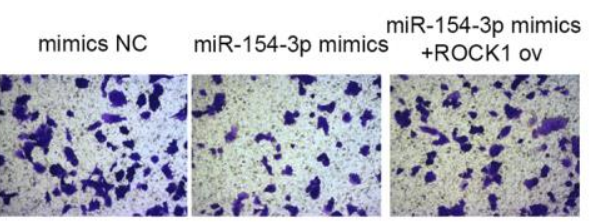

$\mathbf{F}$

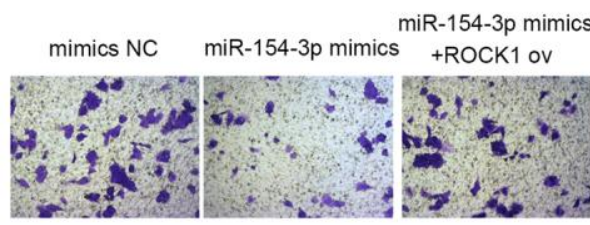

C

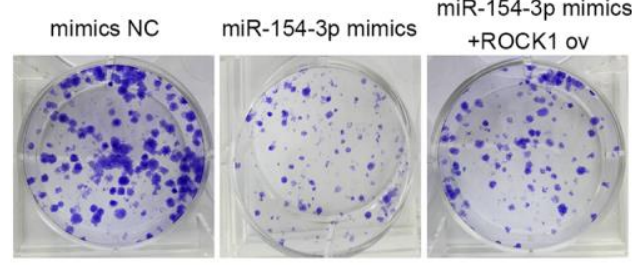

D
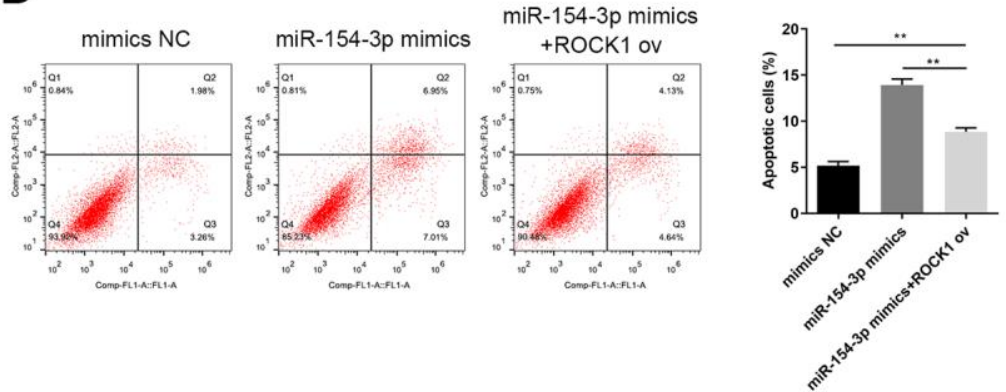

G
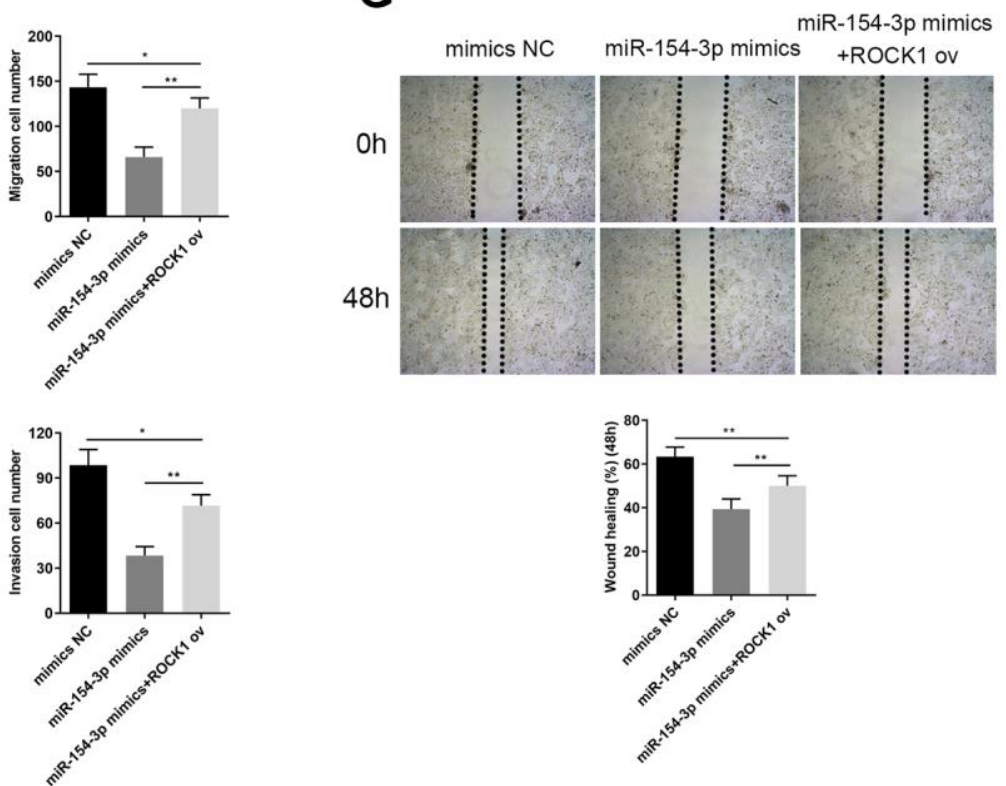

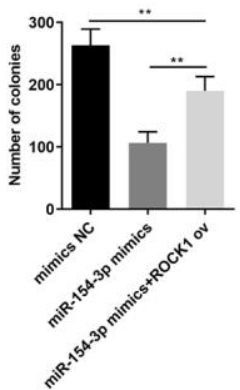

sit

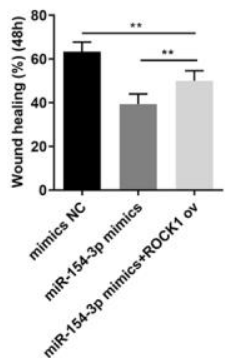

Figure 8. Overexpression of ROCK1 altered changes in cell viability, apoptosis, colony formation ability, migration and invasion ability, caused by miR-154-3p mimics. (A) qRT-PCR assay for ROCK1 mRNA expression in HepG2 cells, transfected with miR154-3p mimics and with or without ROCK1 ov. (B) Cell viability assay was carried out in HepG2 cells transfected with miR-154-3p mimics and with or without ROCK1 ov. (C) Colony formation ability of HepG2 cells that were transfected with miR-154-3p mimics and with or without ROCK1 ov. Quantitative analysis was performed of the colonies were formed (right panel). (D) Apoptosis assays of HepG2 cells transfected with either miR-154-3p mimics and with or without ROCK1 ov, and quantitative analysis was performed of the apoptosis cells (right panel). (E, F) The migration and invasion ability of HepG2 cells transfected with miR-154-3p mimics and with or without ROCK1 ov were determined using the transwell migration assay (E) and transwell invasion assay (F) (left panel). Quantitative analysis of the migratory or invasive cells in transwell assays was also performed (right panel). (G) Migratory ability of HepG2 cells transfected with miR-154-3p mimics and with or without ROCK1 ov at indicated time points, as evaluated by wound healing assay (upper panel). Quantitative analysis of the gap of wound healing assay (lower panel) was performed at 48 hours. ${ }^{*} p<0.05$. 
Linc00205 affects $\mathrm{HB}$ cell malignancy by modifying p38 MAPK signaling pathway-related protein through regulating ROCK1 and may further affect related proteins of the EMT pathway, apoptosis pathways, and cell cycle

Next, we needed to investigate the downstream pathways that were affected by dysregulation of Linc00205. Thus, western blot assays were performed to evaluate the protein expression of several proteins involved in cellular functions. The results demonstrated that the phosphorylation of p38 was impaired when Linc00205 was knocked down, and upregulated when Linc00205 was overexpressed (Figure 9). In addition, p38 is an essential member of the p38 MAPK signaling pathway [20]. Twist1 expression and EMT pathwayrelated protein Vimentin [21] were positively correlated with Linc00205 expression. The expression of cell cycle-related proteins, including cyclinD1 [22], was positively correlated with Linc00205, while expression of Bax, the apoptosis pathway-related protein [23], was negatively correlated with Linc00205 expression. Besides, the western blot results revealed that miR-154$3 p$ inh. could rescue the effect induced by si-Linc00205 on these proteins, and ROCK1 ov could impaired the effect of miR-154-3p mimics. All results indicated that regulating Linc00205 can interact with miR-154-3p and ROCK1 to help produce a massive impact on tumor progression and metastasis through the MAPK signaling pathway, EMT pathway, cell cycle-related pathway and apoptosis-related pathway.

\section{DISCUSSION}

$\mathrm{HB}$ is a common malignant pediatric liver tumor, and the prognosis of $\mathrm{HB}$ varies according to different categories. The practical methods for treating HB are limited, and mainly include surgery and chemotherapy. However, HB has significant tumor heterogeneity, as it is formed by tumors that are derived from different immature liver precursors, including biliary, hepatocytes, and other mesenchymal or epithelial cells [24]. Therefore, it is essential to explore deeper into the underlying molecular mechanism of HB. Herein, we, for the first time, identified Linc00205 as an oncogene of $\mathrm{HB}$ that promotes HB malignancy by regulating metabolic pathways. The Linc00205/miR-154-3p/ROCK1 axis represents a novel target for HB-targeted therapy.

LncRNAs have attracted a lot of attention in recent years for their potential in lncRNA-based cancer therapy. Cancer treatments that target lncRNAs are thought to be novel and promising therapy with high specificity, collective therapeutic efficacy, as well as fewer side effects [25]. Several studies have suggested that dysregulated expression of lncRNAs can be used as novel molecular biomarkers in cancer diagnosis and prognosis prediction [26, 27]. Herein, we identified that Linc00205 is an oncogene, is significantly highly expressed in HB tissues and cells, and high Linc00205 levels are negatively correlated with a five-year survival rate. Furthermore, knockdown of Linc00205 expression contributes to inhibition of $\mathrm{HB}$ cell proliferation, migration and invasion in vitro, as well as reducing tumor volumes in vivo.

It also has been widely accepted that lncRNAs can act as a miRNA sponge, and form a lncRNA-miRNA-mRNA network with miRNAs in order to regulate target protein expression [28]. For example, the LncRNA SPRY4-IT1 can sponge miR-101-3p, and therefore up-regulate EZH2 expression, which promotes proliferation and metastasis of bladder cancer cells [29]. This study shows that Linc00205 sponges miR-154-3p in order to promote proliferation and metastasis of $\mathrm{HB}$ cells by upregulating ROCK1.

The prominent role of the ROCK1 pathway is to regulate cell movement. ROCK1 activation increases phosphorylation of the myosin light chain (MLC) of myosin II, which ultimately increases cell contractility. ROCK1 also participates in phosphorylation of LIMK1/2, which can help increase LIMK catalytic activity and facilitate re-arrangement of the actin cytoskeleton [30]. ROCK1 also participates in regulating cell cycle-related proteins that increase expression of cyclins A/D1/D3 [31], where regulation of cyclin D is thought to be indirectly regulated by activation of Ras/MAPK pathway [32]. The interaction between ROCK1 and MAPK signaling has also been reported by several studies. For example, regulation of RhoC/ ROCK1/MAPK axis likely alters breast cancer cell progression [33]. The LncRNA AFAP1-AS1 promotes tumorigenesis and epithelial-mesenchymal transition of osteosarcoma through the RhoC/ROCK1/p38MAPK/ Twist1 signaling pathway [34]. The MAPK pathway is an important nodal pathway point that the signaling node not only functions as a tumor suppressor, but also as a pro-oncogenic signal. The p38 $\gamma$ MAPK pathway has been suggested to mediate EMT of breast cancer through the p38 $\gamma$ MAPK/GATA3/miR-200b/Suz12 [35]. Translocation of Bax to the mitochondria also requires activation of p38 MAPK, which causes apoptosis [36]. Therefore, cell cycle, apoptosis and EMT pathways are likely all downstream regulatory targets of the MAPK pathway. Herein, ROCK1 was considered to be a target of miR-154-3p, and showed that ROCK1 expression is positively correlated to Linc00205 levels in HB. Our results also demonstrated that Linc00205 facilitates HB progression by competitively binding miR-154-3p in order to activate ROCK1-mediated MAPK pathway, in order to promote EMT. 


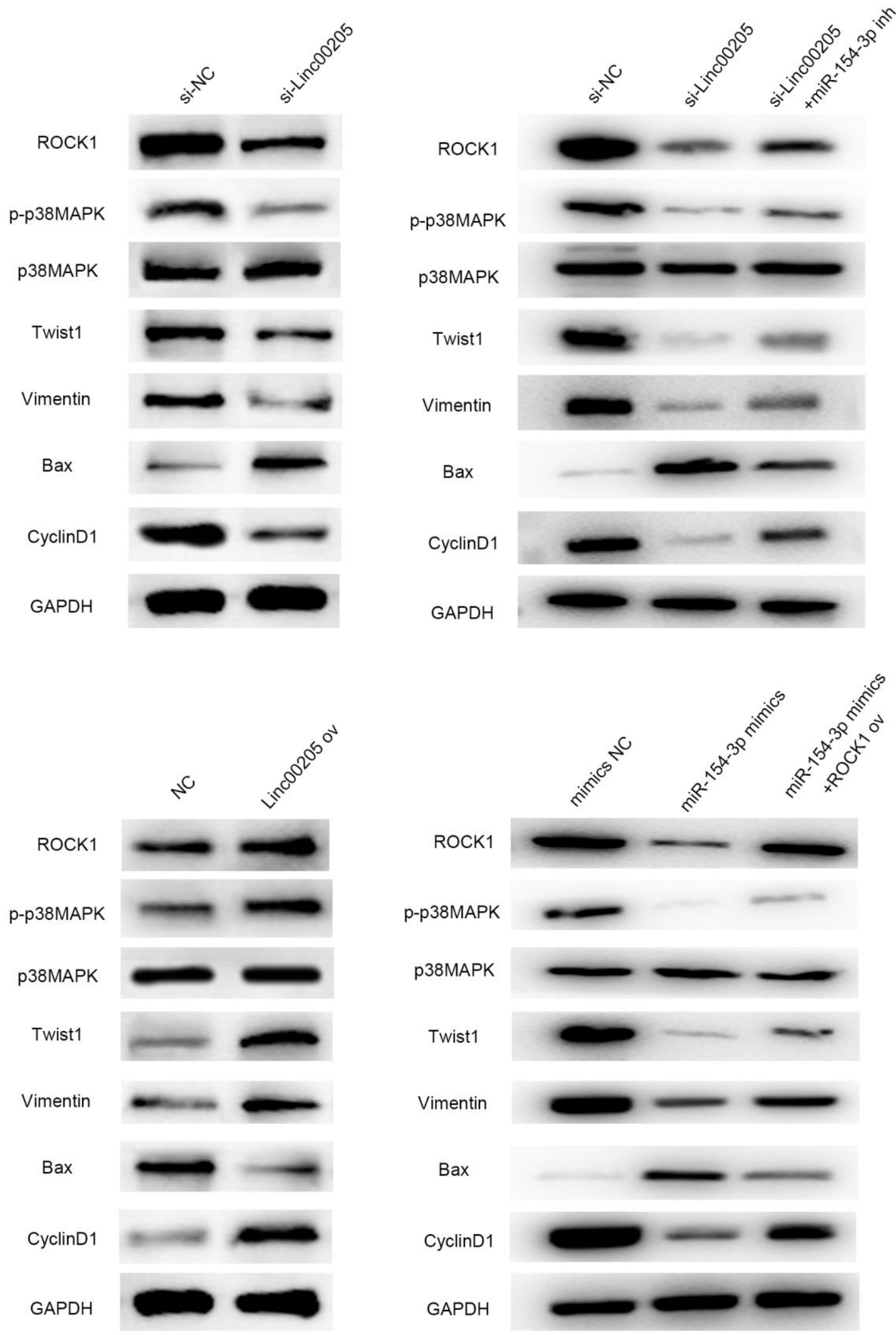

Figure 9. Linc00205 affects the p38 MAPK signaling pathway by interacting with ROCK1, and further affects EMT pathwayrelated protein (vimentin), apoptosis pathway protein (Bax) and proliferation pathway protein (cyclinD1) and the effects are regulated by miR-154-3p and ROCK1. Western blot assays for ROCK1, p-p38MAPK, p38MAPK, Twist, Vimentin, Bax and CyclinD1 protein expression in HepG2 cells transfected in different groups. 


\section{CONCLUSIONS}

In conclusion, we identified Linc00205 as an oncogene that had a crucial role in the proliferation and metastasis in HB. Additionally, our study identified a Linc00205/ miR-154-3p/ROCK1 axis for the first time. We also revealed that Linc00205 positively regulated posttranscriptional expression of ROCK1 by sponging miR-154-3p in HB. Furthermore, knockdown of Linc00205 attenuated oncogenic function and decreased ROCK1 expression, which led to an inhibition of the MAPK pathway, thereby providing a novel therapeutic target in HB. Clinically, the detection and regulation of the Linc00205/miR-154-3p/ROCK1/MAPK axis may play an important role in diagnosis and treatment of HB.

\section{MATERIALS AND METHODS}

\section{Patient samples}

In total, 60 pairs of $\mathrm{HB}$ tissues and para-cancerous tissues were collected from $\mathrm{HB}$ patients at the Children's Hospital of Nanjing Medical University. The inclusion criteria comprised of (1) patients must be diagnosed with $\mathrm{HB}$ with relevant clinical diagnosis, and (2) patients must not have received any anti-tumor treatment prior to surgery. The exclusion criteria indicate that patients with organic diseases and other tumors were excluded. After extraction, the tissues were frozen and stored in a $-80^{\circ} \mathrm{C}$ freezer till the experiments were carried out. All related patients were informed about the research, and signed informed consent letters. The experiments were conducted in agreement with the ethical standards at the ethics committee of Children's Hospital of Nanjing Medical University, as well as the 1964 Helsinki Declaration.

\section{Cell lines and cell culture}

The HB cell lines, including HepG2, SMMC-7721, Huh-6, and the normal human hepatic cell line L02 were cultured in 10\% FBS DMEM medium in an environment of $5 \% \mathrm{CO}_{2}$ and $37^{\circ} \mathrm{C}$.

\section{Cell transfection}

Small interfering RNAs against Linc00205 (siLinc00205), as well as small interfering RNA negative control (si-NC), were cloned into PGK plasmids and used to knockdown expression of Linc00205 in the HepG2 cell line. The pcDNA 3.1 plasmids with full length Linc00205 (Linc00205 ov) and related negative controls (NC) were utilized to upregulate Linc00205 in the Huh-6 cell line. The miR-154-3p inhibitor and mimics were also utilized to regulate expression of miR-154-3p. The pcDNA3.1 plasmids with full length of ROCK1 (ROCK1 ov) and related negative control (NC) were utilized to upregulate ROCK1 in HepG2 cell line. All factors were transfected into cells using Lipofectamine ${ }^{\mathrm{TM}}$ 2000, and adhered to the provided protocol.

\section{Quantitative real-time PCR (qRT-PCR)}

Total RNA in HB tissues and cells was extracted using the TRIzol reagent. RNA was reverse-transcribed into cDNA. Linc00205 and ROCK1 expression were detected using ABI 7900HT RealTime PCR System through the use of SYBR Green assays. GAPDH was used as internal control. The expression of miR-154-3p was quantified via TaqMan MicroRNA Assays and U6 was treated as internal control. The $2^{-\Delta \mathrm{Ct}}$ and $2^{-\Delta \Delta \mathrm{Ct}}$ method helped evaluate RNA expression.

\section{Western blot}

Whole-cell lysate was prepared using the RIPA protein solubilization buffer. Protein levels were quantified utilizing the BCA kits and blocked using 5\% skim milk at room temperature for one hour. After washing with PBS three times, the proteins were incubated with primary antibodies (ROCK1, p-p38MAPK, p38MAPK, Twist, Vimentin, Bax, cyclinD1, and GAPDH) overnight at $4^{\circ} \mathrm{C}$. The membrane was then washed with PBS three times for 5 minutes each time, which was followed by incubation with secondary antibody (1:2000) and incubation at $25^{\circ} \mathrm{C}$ for 2 hours. Finally, the band was visualized using ECL luminescence solution in a dark room. Images were developed and analyzed using the ChemiDocXRS+ system.

\section{Cell proliferation detection}

Cell viability assay was conducted using the Cell Counting Kit-8 (CCK-8), which followed manufacturer's instructions. Results were determined by measuring light absorbance at $450 \mathrm{~nm}$ on a microplate reader. Colony formation assay was carried out by culturing $500 \mathrm{HB}$ transfected cells in 6-well plates, respectively, for two weeks. The colonies were then fixed using $4 \%$ paraformaldehyde for 20 minutes, and then stained with $0.1 \%$ crystal violet for 30 minutes. Finally, the colonies were quantified using the Image $\mathbf{J}$ software.

\section{Cell migration and invasion}

Transwell assay was utilized to determine cell migration and invasion ability of HB cell lines. Cells were placed into the top chamber of a transwell with serum-free media. At the same time, the lower chamber was filled with complete medium. The filters that were used for invasion assays were then coated with $30 \mu \mathrm{L}$ pre-diluted Matrigel. After 24 hours of incubation at $37^{\circ} \mathrm{C}$, the 
migrated or invaded cells were stained using $0.1 \%$ crystal violet, and then observed under a microscope. Cells that migrated into the lower chamber were visualized and counted.

Wound healing assay was utilized to determine the cell migration ability of HB cell lines. A $200 \mu \mathrm{L}$ pipette tip helped create a scraped area in a transfected cells culture dish. Next, cells were washed twice and cultured in medium with $0.1 \%$ FBS in order to eliminate cell proliferation effect. The healing distance was photographed at 0 and 48 hour time points.

\section{Cell apoptosis evaluation}

Cell apoptosis was detected via Annexin V-FITC/PI double staining, and analyzed using flow cytometry. Cells were harvested and washed with PBS, and then resuspended in $500 \mu \mathrm{L}$ binding buffer, which contained $5 \mu \mathrm{L}$ of Annexin V-FITC and $5 \mu \mathrm{L}$ of propidium iodide. After 10 minutes of incubation in the dark at room temperature, samples were then applied for flow cytometry (FCM) analysis.

\section{Dual-luciferase reporter assay}

HepG2 cells were seeded onto 48-well plates until they reached $70 \%$ confluence. Each well was transfected using $100 \mathrm{ng}$ luciferase vector, $400 \mathrm{ng}$ miR-154-3p mimics or mimics NC, and pGL3-Linc00205-wt or pGL3-Linc00205-mut plasmid, pGL3-ROCK1-wt or pGL3-ROCK1-mut plasmid. After the cells were cultured for 24 to 48 hours, cells were washed twice with PBS. With the dual-luciferase reporter assay kit's material and protocol, cells were lysed with $150 \mu \mathrm{L}$ lysate. In addition, $30 \mu \mathrm{L}$ cell lysate was applied to the special black enzyme-labeled hole with addition of LAR II detection buffer for luminescence detection. After adding detection termination solution to each well, luminescence detection was carried out again. Finally, the values were derived and calculated.

\section{Xenograft subcutaneous tumor formation assay}

Five-week old male BALB/C mice were stored at 22$24^{\circ} \mathrm{C}$ routinely and administered dorsal subcutaneous injection of HepG2 cells transfected with si-Linc00205 or Huh- 6 cells that were transfected with Linc00205 ov, suspended in $100 \mu \mathrm{L}$ of Matrigel. Tumor volume was examined every seven days. The calculation formula was as follows: volume $=0.5 \times$ length $\times$ width $\times$ height . Then, five weeks after the injection, animals were euthanized and their tumor weights were measured. Animal experiments were granted approval by the Animal Ethics Committee of Children's Hospital of Nangjing Medical University.

\section{Statistical analysis}

Statistical analysis was carried out using the SPSS software (Version 20.0) and graphpad7. Data were presented as mean \pm standard deviation $(\mathrm{x} \pm \mathrm{s})$ of at least three biological replicates. A $t$-test was utilized for the intergroup comparisons, and a one-way analysis of variance was used to compare between groups. Values of $\mathrm{p}<0.05$ indicated statistical significance.

\section{AUTHOR CONTRIBUTIONS}

Guoqing Liu and Qiang Zhu performed the experiments and wrote the draft; Hao Wang and Jianfeng Zhou analyzed the data; Bin Jiang designed and supervised the study, and polished the draft.

\section{CONFLICTS OF INTEREST}

The authors declare that they have no conflicts of interest.

\section{FUNDING}

This study was funded by Natural Science Foundation of Jiangsu Province (No. BK20190138).

\section{REFERENCES}

1. Feusner J, Buckley J, Robison L, Ross J, Van Tornout J. Prematurity and hepatoblastoma: more than just an association? J Pediatr. 1998; 133:585-6. https://doi.org/10.1016/s0022-3476(98)70084-8 PMID: 9787710

2. Ranganathan S, Lopez-Terrada D, Alaggio R. Hepatoblastoma and Pediatric Hepatocellular Carcinoma: An Update. Pediatr Dev Pathol. 2020; 23:79-95. https://doi.org/10.1177/1093526619875228 PMID: 31554479

3. Herzog CE, Andrassy RJ, Eftekhari F. Childhood cancers: hepatoblastoma. Oncologist. 2000; 5:445-53. https://doi.org/10.1634/theoncologist.5-6-445 PMID:11110595

4. Meyers RL, Maibach R, Hiyama E, Häberle B, Krailo M, Rangaswami A, Aronson DC, Malogolowkin MH, Perilongo $G$, von Schweinitz D, Ansari M, LopezTerrada D, Tanaka $Y$, et al. Risk-stratified staging in paediatric hepatoblastoma: a unified analysis from the Children's Hepatic tumors International Collaboration. Lancet Oncol. 2017; 18:122-31. https://doi.org/10.1016/S1470-2045(16)30598-8 PMID:27884679 
5. Deniz E, Erman B. Long noncoding RNA (lincRNA), a new paradigm in gene expression control. Funct Integr Genomics. 2017; 17:135-43.

https://doi.org/10.1007/s10142-016-0524-x

PMID:27681237

6. Yan X, Hu Z, Feng Y, Hu X, Yuan J, Zhao SD, Zhang Y, Yang L, Shan W, He Q, Fan L, Kandalaft LE, Tanyi JL, et al. Comprehensive Genomic Characterization of Long Non-coding RNAs across Human Cancers. Cancer Cell. 2015; 28:529-40.

https://doi.org/10.1016/i.ccell.2015.09.006

PMID:26461095

7. Xie P, Guo Y. LINC00205 promotes malignancy in lung cancer by recruiting FUS and stabilizing CSDE1. Biosci Rep. 2020; 40:BSR20190701.

https://doi.org/10.1042/BSR20190701

PMID:32808651

8. Wahid F, Shehzad A, Khan T, Kim YY. MicroRNAs: synthesis, mechanism, function, and recent clinical trials. Biochim Biophys Acta. 2010; 1803:1231-43. https://doi.org/10.1016/i.bbamcr.2010.06.013 PMID:20619301

9. Cartier F, Indersie E, Lesjean S, Charpentier J, Hooks KB, Ghousein A, Desplat A, Dugot-Senant N, Trézéguet V, Sagliocco F, Hagedorn M, Grosset CF. New tumor suppressor microRNAs target glypican-3 in human liver cancer. Oncotarget. 2017; 8:41211-26.

https://doi.org/10.18632/oncotarget.17162 PMID:28476031

10. Ren $H$, Ma X, Shao $Y$, Han J, Yang $M$, Wang $Q$. Correlation Between Serum miR-154-5p and Osteocalcin in Males and Postmenopausal Females of Type 2 Diabetes With Different Urinary Albumin Creatinine Ratios. Front Endocrinol (Lausanne). 2019; 10:542.

https://doi.org/10.3389/fendo.2019.00542

PMID: $\underline{31447785}$

11. Dong $\mathrm{P}$, Liu WJ, Wang ZH. MiR-154 promotes myocardial fibrosis through $\beta$-catenin signaling pathway. Eur Rev Med Pharmacol Sci. 2018; 22:2052-60.

https://doi.org/10.26355/eurrev 20180414735 PMID:29687862

12. Pang $X$, Huang $K$, Zhang Q, Zhang $Y$, Niu J. miR-154 targeting ZEB2 in hepatocellular carcinoma functions as a potential tumor suppressor. Oncol Rep. 2015; 34:3272-9.

https://doi.org/10.3892/or.2015.4321 PMID:26503460

13. Dong R, Liu GB, Liu BH, Chen G, Li K, Zheng S, Dong KR. Targeting long non-coding RNA-TUG1 inhibits tumor growth and angiogenesis in hepatoblastoma. Cell Death Dis. 2016; 7:e2278. https://doi.org/10.1038/cddis.2016.143

PMID:27362796

14. Zhang L, Wang Y, Sun J, Ma H, Guo C. LINC00205 promotes proliferation, migration and invasion of HCC cells by targeting miR-122-5p. Pathol Res Pract. 2019; 215:152515.

https://doi.org/10.1016/i.prp.2019.152515 PMID:31272761

15. Hartmann S, Ridley AJ, Lutz S. The Function of RhoAssociated Kinases ROCK1 and ROCK2 in the Pathogenesis of Cardiovascular Disease. Front Pharmacol. 2015; 6:276.

https://doi.org/10.3389/fphar.2015.00276 PMID:26635606

16. Lochhead PA, Wickman G, Mezna M, Olson MF. Activating ROCK1 somatic mutations in human cancer. Oncogene. 2010; 29:2591-8. https://doi.org/10.1038/onc.2010.3 PMID:20140017

17. An L, Liu Y, Wu A, Guan Y. microRNA-124 inhibits migration and invasion by down-regulating ROCK1 in glioma. PLoS One. 2013; 8:e69478.

https://doi.org/10.1371/journal.pone.0069478 PMID:23936026

18. Wan X, Cheng Q, Peng R, Ma Z, Chen Z, Cao Y, Jiang B. ROCK1, a novel target of miR-145, promotes glioma cell invasion. Mol Med Rep. 2014; 9:1877-82.

https://doi.org/10.3892/mmr.2014.1982

PMID:24573110

19. Fan XD, Luo Y, Wang J, An N. miR-154-3p and miR-487$3 p$ synergistically modulate RHOA signaling in the carcinogenesis of thyroid cancer. Biosci Rep. 2020; 40:BSR20193158.

https://doi.org/10.1042/BSR20193158

PMID:31820783

20. Zarubin T, Han J. Activation and signaling of the $\mathrm{p} 38$ MAP kinase pathway. Cell Res. 2005; 15:11-8. https://doi.org/10.1038/sj.cr.7290257 PMID:15686620

21. Wang X, Zheng M, Liu G, Xia W, McKeown-Longo PJ, Hung MC, Zhao J. Krüppel-like factor 8 induces epithelial to mesenchymal transition and epithelial cell invasion. Cancer Res. 2007; 67:7184-93. https://doi.org/10.1158/0008-5472.CAN-06-4729 PMID:17671186

22. Alao JP. The regulation of cyclin D1 degradation: roles in cancer development and the potential for therapeutic invention. Mol Cancer. 2007; 6:24. https://doi.org/10.1186/1476-4598-6-24 PMID:17407548

23. Peña-Blanco A, García-Sáez AJ. Bax, Bak and beyond mitochondrial performance in apoptosis. FEBS J. 2018; 285:416-31. 
https://doi.org/10.1111/febs.14186

PMID:28755482

24. Rowland JM. Hepatoblastoma: assessment of criteria for histologic classification. Med Pediatr Oncol. 2002; 39:478-83.

https://doi.org/10.1002/mpo.10171

PMID:12228903

25. Jin SJ, Jin $M Z$, Xia BR, Jin WL. Long Non-coding RNA DANCR as an Emerging Therapeutic Target in Human Cancers. Front Oncol. 2019; 9:1225. https://doi.org/10.3389/fonc.2019.01225 PMID:31799189

26. Hu Y, Chen HY, Yu CY, Xu J, Wang JL, Qian J, Zhang X, Fang JY. A long non-coding RNA signature to improve prognosis prediction of colorectal cancer. Oncotarget. 2014; 5:2230-42.

https://doi.org/10.18632/oncotarget.1895

PMID:24809982

27. Meng J, Li P, Zhang Q, Yang Z, Fu S. A four-long noncoding RNA signature in predicting breast cancer survival. J Exp Clin Cancer Res. 2014; 33:84.

https://doi.org/10.1186/s13046-014-0084-7 PMID:25288503

28. Salmena L, Poliseno L, Tay Y, Kats L, Pandolfi PP. A ceRNA hypothesis: the Rosetta Stone of a hidden RNA language? Cell. 2011; 146:353-8.

https://doi.org/10.1016/i.cell.2011.07.014

PMID:21802130

29. Liu D, Li Y, Luo G, Xiao X, Tao D, Wu X, Wang M, Huang $C$, Wang L, Zeng F, Jiang G. LncRNA SPRY4-IT1 sponges miR-101-3p to promote proliferation and metastasis of bladder cancer cells through up-regulating EZH2. Cancer Lett. 2017; 388:281-91. https://doi.org/10.1016/i.canlet.2016.12.005 PMID:27998761

30. Chen W, Mao K, Liu Z, Dinh-Xuan AT. The role of the RhoA/Rho kinase pathway in angiogenesis and its potential value in prostate cancer (Review). Oncol Lett. 2014; 8:1907-11. https://doi.org/10.3892/ol.2014.2471 PMID:25289078
31. Street CA, Bryan BA. Rho kinase proteins--pleiotropic modulators of cell survival and apoptosis. Anticancer Res. 2011; 31:3645-57.

PMID:22110183

32. Croft DR, Olson MF. The Rho GTPase effector ROCK regulates cyclin A, cyclin D1, and p27Kip1 levels by distinct mechanisms. Mol Cell Biol. 2006; 26:4612-27. https://doi.org/10.1128/MCB.02061-05 PMID:16738326

33. Rosenthal DT, lyer $\mathrm{H}$, Escudero $\mathrm{S}$, Bao L, Wu Z, Ventura AC, Kleer CG, Arruda EM, Garikipati K, Merajver SD. p38y promotes breast cancer cell motility and metastasis through regulation of RhoC GTPase, cytoskeletal architecture, and a novel leading edge behavior. Cancer Res. 2011; 71:6338-49.

https://doi.org/10.1158/0008-5472.CAN-11-1291 PMID:21862636

34. Shi D, Wu F, Mu S, Hu B, Zhong B, Gao F, Qing X, Liu J, Zhang Z, Shao Z. LncRNA AFAP1-AS1 promotes tumorigenesis and epithelial-mesenchymal transition of osteosarcoma through RhoC/ROCK1/p38MAPK/ Twist1 signaling pathway. J Exp Clin Cancer Res. 2019; 38:375.

https://doi.org/10.1186/s13046-019-1363-0 PMID:31443665

35. Xu M, Wang S, Wang $\mathrm{Y}$, Wu H, Frank JA, Zhang Z, Luo J. Role of p38y MAPK in regulation of EMT and cancer stem cells. Biochim Biophys Acta Mol Basis Dis. 2018; 1864:3605-17.

https://doi.org/10.1016/j.bbadis.2018.08.024 PMID:30251680

36. Van Laethem A, Van Kelst S, Lippens S, Declercq W, Vandenabeele P, Janssens S, Vandenheede JR, Garmyn $\mathrm{M}$, Agostinis P. Activation of p38 MAPK is required for Bax translocation to mitochondria, cytochrome $c$ release and apoptosis induced by UVB irradiation in human keratinocytes. FASEB J. 2004; 18:1946-8. https://doi.org/10.1096/fj.04-2285fje PMID: 15388671 